\title{
CIII. Initial stages of ionization by collision
}

\section{S. Smith B.A.}

To cite this article: S. Smith B.A. (1914) CIII. Initial stages of ionization by collision, Philosophical Magazine Series 6, 27:162, 963-976, DOI: 10.1080/14786440608635166

To link to this article: http://dx.doi.org/10.1080/14786440608635166

曲 Published online: 08 Apr 2009.

Submit your article to this journal 준

Џll Article views: 2

Q View related articles $₫$ 
Quantitative relationships between partial prossure and freezing-point data may readily be obtained from equations (28), (70), and (71). Thus we have

$$
\begin{aligned}
& \frac{\theta}{\mathrm{T}^{2}} \frac{\partial \mathrm{T}}{\partial s_{i}}\left[\mathrm{~L}_{0}-l_{0}-\gamma\left(\mathrm{T}_{0}-\mathrm{T}\right)\right]+\frac{\theta-\mathrm{T}}{\mathrm{T}} \frac{\partial l_{0}}{\partial s_{i}}=\mathrm{V}_{1}\left(\Pi_{0}, \theta\right) \frac{\partial \Pi_{0}}{\partial s_{i}} \\
& \text { The University, Leeds, } \\
& \ldots(i=1,2) \text {. . . . }
\end{aligned}
$$

Sept. 18, 1913.

CIII. Initial Stages of Ionization by Collision. By S. Sмiтн, B.A., Exeter Ciollege, Oxford*.

W HEN negative ions are liberated from a narrow electrode such as $O$ (fig. 1), and made to move in the electronic state under the influence of a uniform electric field

Fig. 1.
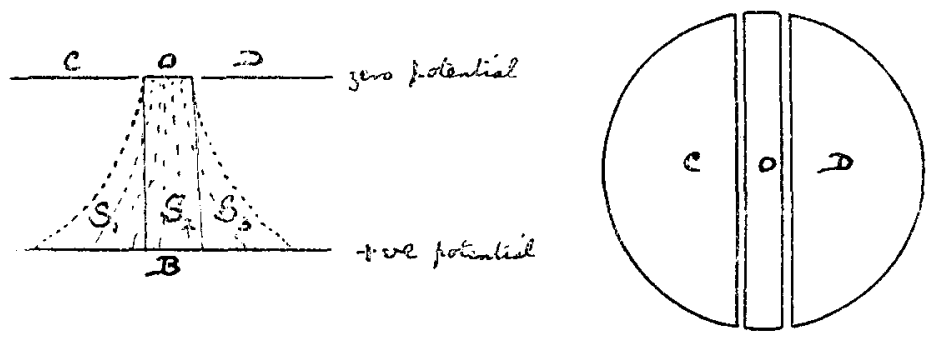

between parallel-plate electrodes to $B$, there is found to be a considerable spreading of the beam. The amount of this spreading depends on the ratio of the applied electric force, $Z$, to a quantity $k, k$ being the ratio of the partial pressure of the ions to that of an equal number of molecules of the surrounding gas $\dagger$. When the ratio of the electric force $Z$ to the pressure $p$ of the gas becomes sufficiently large, the negative ions produce ions in the gas by collisions. The positive ions thus produced possess at least atomic masses : their lateral diffusion may therefore be neglected, since $Z$ is larue. Hence all the positive ions produced in the spaces $S_{1}, S_{2}$, and $S_{3}$ reach the portions $\mathrm{C}, \mathrm{O}$, and $\mathrm{D}$, respectively, of the top electrode; $O$ being separated from $C$ and $D$ by very narrow spaces. It is assumed that $\frac{Z}{p}$ is so small that the positive ions do not produce any ions by collisions. The

* Communicated by Prof. J. S. Townsend, F.R.S.

+ Prof. Townsend, Proc. Roy. Soc. A. vol. lxxxi. (1908). 
positive charges which arrive at $\mathrm{C}, \mathrm{O}$, and $\mathrm{D}$ are therefore proportional to the number of molecules ionized by negative ions in the spaces $S_{1}, S_{2}$, and $S_{3}$ respectively. It is clear that with an apparatus designed on these principles one could detect the first indications of the production of ions by collisions for small values of $\frac{\mathrm{Z}}{p}$.

The ratio, $\mu$, of the number of negative ions produced by collisions in $\mathrm{S}_{2}$ to the total number produced by collisions can be found theoretically for the different values of $\frac{Z}{p}$. It will be seen that for the larger values of $\alpha$ ( $\alpha$ being the number of negative ions produced by collision by a negative ion whilst moving through a distance of one centimetre in the direction of the electric field) the ratio $\mu$ is a function of $\alpha$, but for the smalier values of $\alpha, \mu$ is independent of $\alpha$. The latter is the more important case, for by substituting the theoretical value of $\mu$ in expressions found from experimental measurements values of $\alpha$ can be deduced.

In the experiments the stream of negative ions was obtained by allowing ultra-violet light to fall on the electrode $O$, and as it was of great importance that the ions should be given off uniformly over the electrode, a preliminary experiment was made on the silver plate used in the apparatus. The plate and $a$ sheet of copper gauze were used as parallel electrodes, and a narrow beam of ultraviolet light was allowed to fall on different parts of the plate. The saturation currents between the electrodes were found to be very approximately the same in every case, showing that the photoelectric effect was uniform over the plate.

The top electrodes 1,2 , and 3 (fig. 2) were cut from this plate, which was $4.5 \mathrm{~cm}$. in diameter. The width of electrode 2 is $4 \mathrm{~mm}$., and electrodes 1 and 3 are separated from electrode 2 by gaps of $\frac{1}{2} \mathrm{~mm}$. each. The bottom electrode, $B$, which is situated $2 \mathrm{~cm}$. from electrodes 1,2 , and 3 and parallel to them, is a silvered quartz plate on which is ruled a grating of length $15 \mathrm{~mm}$. and width $3 \mathrm{~mm}$., the lines of the grating being equally spaced at distances of $\frac{1}{2} \mathrm{~mm}$. B fits into a brass ring, which is supported by brass pillars fixed to the base $F$. When $B$ is in position the grating is parallel to electrode $2 . \mathrm{G}$ is a brass guard-ring. The pillars $\mathrm{P}, \mathrm{P}$, the block $\mathrm{A}$, and the plugs $\mathrm{C}$ and $\mathrm{D}$ are all of ebonite. In the brass baso is a rectangular gap, $\mathrm{S}$, of length $1.5 \mathrm{~cm}$. and width $3 \mathrm{~mm}$. parallel to electrode 2 , and the apparatus was so made that the line of centres of electrode 2, of the grating, and of the gap is normal to the 
planes of the electrodes. $B$ is a quartz plate of diameter $4.5 \mathrm{~cm}$. The bell-jar, $J$, was so affixed as to render the apparatus air-tight. $J$ was attached to drying-tubes, a Toepler pump, a MacLeod gauge, a pressure adjustor, and a reservoir containing a drying agent.

Fig. 2.

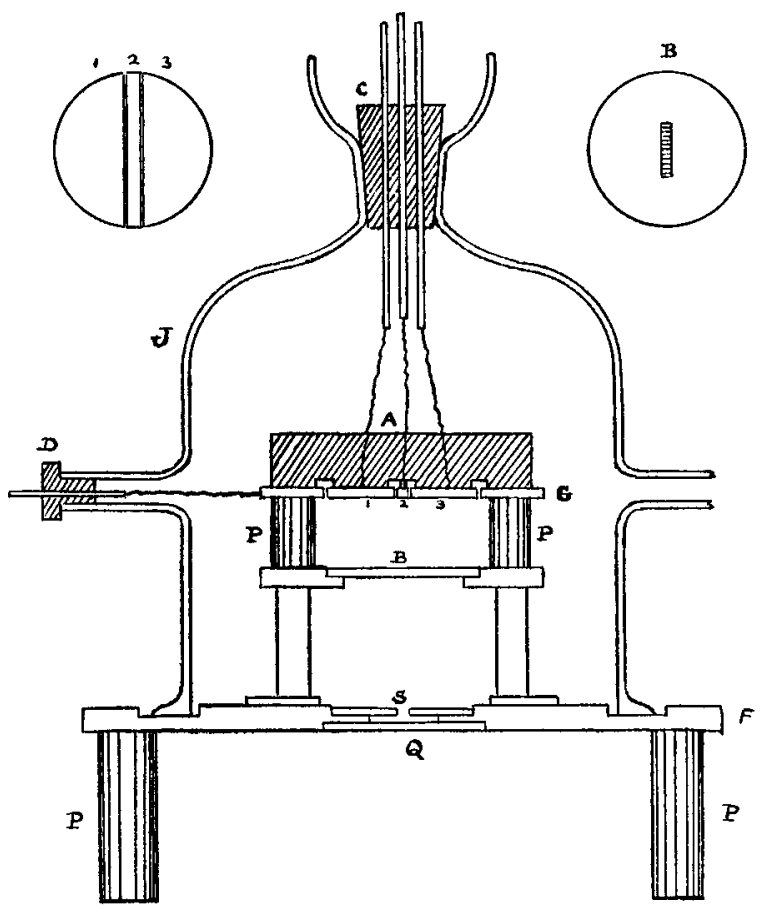

The apparatus was made in the laboratory by Mr. Bush, to whom I should like to take this opportunity of expressing my indebtedness for the accuracy shown in the construction.

The ultra-violet light was obtained from an aluminium spark-gap of length $3.5 \mathrm{~mm}$. placed $6 \mathrm{~cm}$. vertically below the centre of $G$, the length of the spark-gap being at right angles to the length of electrode 2. The Rubmkorff coil and spark-gap were enclosed in an earthed lead box with a suitable aperture above the spark-gap.

Let $q_{0}$ be the positive charge left on electrode 2 due to the liberation of negative ions by the ultra-violet light, and let $q_{1}, q_{2}$, and $q_{3}$ be the positive charges which arrive at electrodes 1,2 , and 3 respectively, as a result of the ionization

Phil. Mag. S. 6. Vol. 27. No. 162. June 1914. 3 S 
by collisions in the spaces $S_{1}, S_{2}$, and $S_{3}$. The total charge on 2 is $q_{0}+q_{2}$. The terminals of electrodes 1 and 3 were joined, and $\frac{q_{1}+q_{3}}{q_{0}+q_{2}}$ was measured experimentally. For this purpose the method which is in common use in the laboratory for measuring the ratio of two charges was adopted. The arrangement is shown in fig. 3.

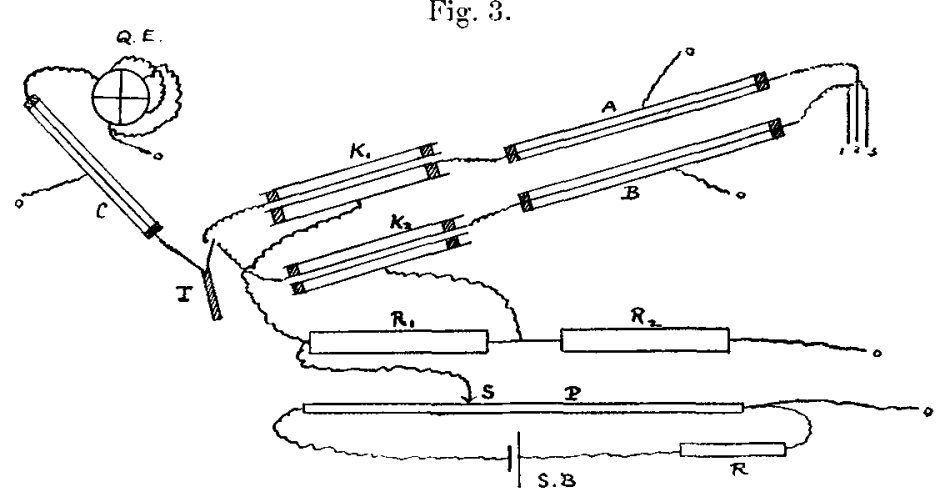

$\mathrm{A}, \mathrm{B}$, and $\mathrm{C}$ are earthed cylindrical tubes, fitted with ebonite plugs, to guard the leading wires from induction effects. $K_{1}$ and $K_{2}$ are cylindrical condensers of equal capacity, C. Q.E. is a Dolezalek electrometer, T a key enabling the electrometer to be put into connexion with the inside cylinder of either $K_{1}$ or $K_{2} . \quad R_{1}, R_{2}$, and $R$ are resistance-boxes, $P$ a potentiometer, and S.B. a storagebattery.

The positive charge gained by the electrode 2 tends to increase the potential of the inside cylinder of condenser $K_{1}$, and this can be neutralized by bringing the outside cylinder to a certain negative potential V.

Then

$$
q_{0}+q_{2}=-\mathrm{V} . \mathrm{C} \text {. }
$$

Also, by adjusting the ratio of the resistance $R_{1}$ to the resistance $\mathrm{R}_{2}$, the inside cylinder of condenser $\mathrm{K}_{2}$ can at the same time be kept at zero potential. The cuter cylinder of $\mathrm{K}_{2}$ is thus raised to potential $\frac{R_{2}}{R_{1}+R_{2}} \cdot V$;

hence

$$
\begin{aligned}
& q_{1}+q_{3}=-\frac{\mathrm{R}_{2}}{\mathrm{R}_{1}+\mathrm{R}_{2}} \cdot V, \\
& \frac{q_{1}+q_{3}}{q_{0}+q_{2}}=\frac{\mathrm{R}_{2}}{\mathrm{R}_{1}+\mathrm{R}_{2}}=\rho .
\end{aligned}
$$


In order to obtain a measurement of $\rho$ the electrodes 1,2 , and 3 were earthed, and the lower plate was connected to the battery. The earth connexion was then removed and the electrometer was put into connexion with the inside cylinder of $\mathrm{K}_{1}$. The ultra-violet light was turned on for a period of time depending on the magnitude of $\alpha$, and the slider $\mathbf{S}$ was moved until the electrometer needle was brought back to its zero position. This could be done with great accuracy by using the adjustable resistance $\mathrm{R}$. The electrometer was then switched on to the inside cylinder of $\mathrm{K}_{2}$, and $R_{1}$ and $R_{2}$ were adjusted so that the electrometer needle was again brought to the zero position. The ratio $\frac{R_{2}}{R_{1}+R_{2}}$ was taken as a first approximation, and several more readings were taken to obtain an exact value.

The curves in fig. 4 represent the results of the experiments

Fig. 4.

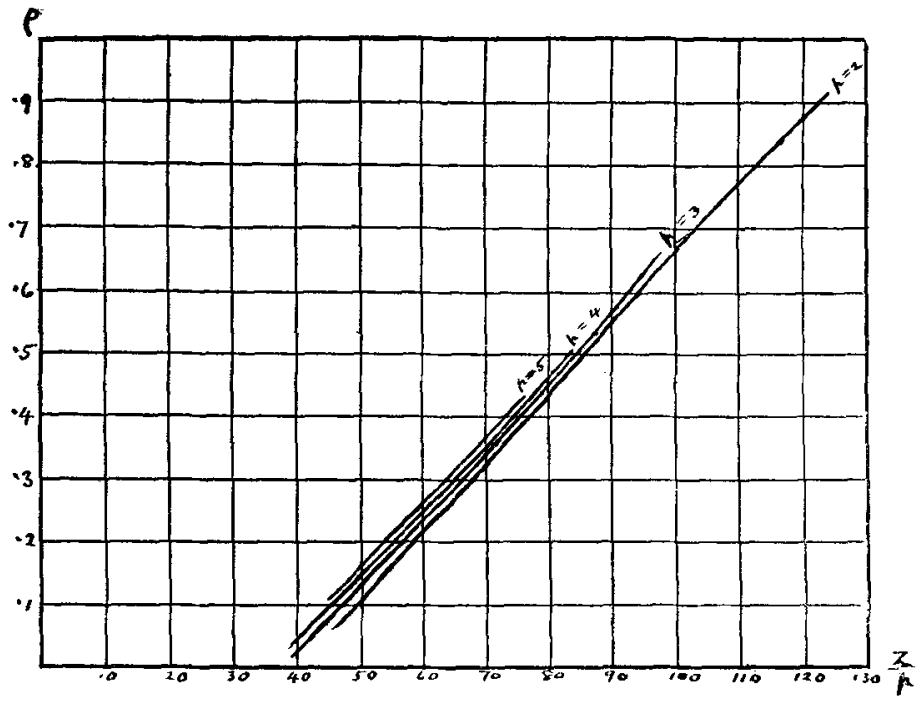

made with air at pressures of $2,3,4$, and $5 \mathrm{~mm}$. of mercury. The air was dried very thoroughly by allowing it to remain in the reservoir for some weeks.

The theory requires that the rays should fall symmetrically on the electrode 2. In order to obtain this condition of symmetry the leads from electrodes 1 and 3 were connected with the inside cylinders of $K_{1}$ and $K_{2}$ respectively, electrode 2 was earthed, and the apparatus was moved (the slightest motion was found to be sufficient) relative to the spark-gap 
to such a position that the charges gathered on electrodes 1 and 3 were equal when the light had been turned on.

In order to obtain an expression for the distribution of negative ions between the electrodes, let the geometrical centre of the face of electrode 2 be taken as the origin of rectangular coordinates, the normal to the face as the axis of $z$, and the axis of $y$ parallel to the length of the electrode. Let $n$ be the number of negative ions per cubic centimetre in the neighbourhood of the point $(x, y, z), \mathrm{Z}$ the electric force at that point in electrostatic units, and $e$ the charge on an ion in electrostatic units. The number of ions crossing per second unit area normal to $Z$, may be represented as $n w$, and is due to the processes of diffusion and motion under the electric force, so that $n w$ is given by the equation

$$
n w=-\mathrm{K} \frac{\partial n}{\partial z}+n w_{1} \mathrm{Z}
$$

where $w_{1}$ is the mobility of the ions or velocity under unit electric force*. This expression holds good as long as the velocity due to the electric force is small compared with $\mathrm{V}$, the mean velocity of agitation. Reference to a paper by Prof. Townsend and H. T. Tizard t will show that this condition has not been violated throughout the experiments.

The velocity of the ions is therefore

$$
(u, v, w) \equiv\left(-\frac{1}{n} \mathrm{~K} \frac{\partial n}{\partial x}, \quad-\frac{1}{n} \mathrm{~K} \frac{\partial n}{\partial y}, \quad-\frac{1}{n} \mathrm{~K} \frac{\partial n}{\partial z}+w_{1} \mathrm{Z}\right) .
$$

Taking a parallelopiped of sides $\delta x, \delta y$, and $\delta z$ about the point $(x, y, z)$, it is clear that the excess of the number of negative ions coming into the parallelopiped per second over those going out per second is given by

$$
\Sigma\left\{n u \delta y \delta z-\left[n u+\frac{\partial}{\partial x}(n u) \delta x\right] \delta y \delta z\right\}
$$

and this is easily seen to be

$$
\left\{\mathrm{K} \nabla^{2}(n)-w_{1} \mathrm{Z} \frac{\partial n}{\partial z}\right\} \delta x \cdot \delta y \cdot \delta z . . \cdot
$$

Now the number of negative ions produced per second by collisions in the parallelopiped is $n w_{1} Z \delta ! \cdot \delta z \cdot a \delta x$. In the steady state the sum of these expressions must be zero, so that

$$
\mathrm{K} \cdot \nabla^{2}(n)-w_{1} \mathrm{Z} \frac{\partial n}{\partial z}+n a w_{1} \mathrm{Z}=0 .
$$

* Prof. Townsend, Proc. Roy. Soc. A. vol. lxxxvi. (1912).

+ Proc. Roy. Soc, A, vol. Ixxxviii. (1913). 
But $\frac{w_{1}}{\mathrm{~K}}=\frac{\mathrm{Ne}}{k \overline{\mathrm{II}}} *$, where $\mathrm{N}$ is the number of molecules per cubic centimetre in the gas at atmospheric pressure $\Pi$ and at the temperature of the room, and $k$ is the factor by which the energy of agitation of an electron exceeds that of a molecule of the gas, $k$ being a function of $\frac{Z}{p}$. Hence (1)
becomes

$$
\nabla^{2}(n)-\gamma \frac{\partial n}{\partial z}+\gamma \cdot \alpha \cdot n=0, . . . \cdot
$$

where

$$
\gamma=\frac{\mathrm{NeZ}}{\mathrm{k} \Pi \text {. }}
$$

The number of negative ions produced by collisions in a period of time $\tau$ is given by an integral of the form

$$
\mathrm{A} \int\left[\iint n d x \cdot d y\right] \alpha d z
$$

where $\mathrm{A}$ involves $w_{1}$ and $\tau$.

Hence the ratio, $\mu$, of the number of ions produced by collision inside the limits $x=\cdot 2 \mathrm{~cm}$. and $x==-\cdot 2 \mathrm{~cm}$. to the total number produced is

$$
\frac{\int_{0}^{+2} d x \int_{0}^{2} d z \int_{-\infty}^{\infty} n d y}{\int_{0}^{\infty} d x \int_{0}^{+2} d z \int_{-\infty}^{\infty} n d y}=\mu
$$

Since the emission of ions is confined to the central portion of electrode 2 , it becomes apparent that for the larger values of $\frac{\mathrm{Z}}{k}$ the ions do not extend to the boundary (which is represented by $\infty$ ). Hence at the boundary $n=0$ and $\frac{\partial n}{\partial y}=0$. Equation (2) on integration with regard to $y$ becomes

$$
\frac{\partial^{2} q}{\partial x^{2}}+\underset{\text { boundary limits }}{\left[\frac{\partial n}{\partial y}\right]}+\frac{\partial^{2} q}{\partial z^{2}}-\gamma \frac{\partial q}{\partial z}+\gamma \cdot \alpha \cdot q=0,
$$

where $q=\int n d y$, the limits of integration being the values of $y$ at opposite points on the boundary. Hence $\left[\begin{array}{l}\partial n \\ \partial y\end{array}\right]=0$.

Also since the ions for the larger values of $\frac{\mathrm{Z}}{k}$ move in a

* Proc. Roy. Soc. A. vol. lxxxi. (1908). 
narrow stream chiefly in the direction of the axis of $z$, $\frac{\partial^{2} q}{\partial z^{2}}$ is small compared with $\frac{\partial^{2} q}{\partial x^{2}}$ or $\gamma \frac{\partial q}{\partial z}$.

Hence neglecting $\frac{\partial^{2} q}{\partial z^{2}}$ the above equation becomes

$$
\frac{\partial^{2} q}{\partial x^{2}}-\gamma \frac{\partial q}{\partial z}+\gamma \cdot \alpha \cdot q=0
$$

or

$$
\frac{\partial^{2} \dot{\phi}}{\partial x^{2}}=\gamma \frac{\partial \phi}{\partial z}
$$

where

$$
q=\phi e^{a z}
$$

The solution is required to satisfy the following boundary conditions:

$$
\left.\begin{array}{l}
\phi=\text { constant from } x=\cdot 2 \text { to } x=-\cdot 2 \mathrm{~cm} . \\
\phi=0 \quad \text { when } x>\cdot 2 \text { or }<-\cdot 2 \mathrm{~cm} .
\end{array}\right\} z=0 .
$$

Hence it is clear that $\phi$ is a quantity which varies with $x$ and $z$ in the same way as the temperature $\theta$ varies with $x$ and the time $t$, in an infinite heat-conducting solid, whose initial condition is that, when $t=0 \theta$ is zero everywhere except in an infinite slab bounded by planes $x=\cdot 2 \mathrm{~cm}$. and $x=-\cdot 2 \mathrm{~cm}$., where the temperature has a uniform value.

Therefore

$$
\phi=\mathrm{A} \int_{-\cdot 2}^{\cdot 2} z^{-\frac{1}{2}} e^{-\frac{\gamma(x-\xi)^{2}}{4 z}} d \xi
$$

and

$$
q=\mathrm{A} e^{\alpha z} \int_{-\cdot 2}^{\cdot 2} z^{-\frac{x}{2}} e^{-\frac{\gamma(x-\xi)^{2}}{4 z}} d \xi
$$

Hence $\mu$ may be found by substituting this value of $q$ in the equation

$$
\mu=\frac{\int_{0}^{\cdot 2} d x \int_{0}^{2} q d z}{\int_{0}^{\infty} d x \int_{0}^{2} q d z}
$$

This ratio is independent of $\alpha$ when $\alpha$ is small. The ratio $\mu$ depends on $\frac{Z}{k}$ since $\gamma=\frac{N e}{\Pi k} Z=41 \cdot \frac{Z}{k}$, where $Z$ is expressed in volts per $\mathrm{cm}$. The values of $k$ corresponding to 
different forces and pressures have been determined. Hence the values of $\mu$ may be represented by a curve in terms of $\frac{Z}{\bar{k}}$, when $\alpha$ is small. The curve is shown in fig. 4 .

The connexion between $\mu$ and the quantity $\rho$, which is determined experimentally, may easily be found. Let $n_{0}$ be the number of ions set free by the photo-electric effect, $n_{1}$, $n_{2}$, and $n_{3}$ the numbers of negative ions generated by collisions in the spaces $S_{1}, S_{2}$, and $S_{3}$ respectively.

Then

$$
\begin{aligned}
& \mu=\frac{n_{2}}{n_{1}+n_{2}+n_{3}}=\frac{q_{2}}{q_{1}+q_{2}+q_{3}}, \\
& \rho=\frac{q_{1}+q_{3}}{q_{0}+q_{2}},
\end{aligned}
$$

also

$$
n_{1}+n_{2}+n_{3}+n_{0}=n_{0} e_{2}^{\circ} .
$$

Hence

$$
e^{2 a}=\frac{(\rho+1)(\mu-1)}{\mu(\rho+1)-1}
$$

Fig. 5.

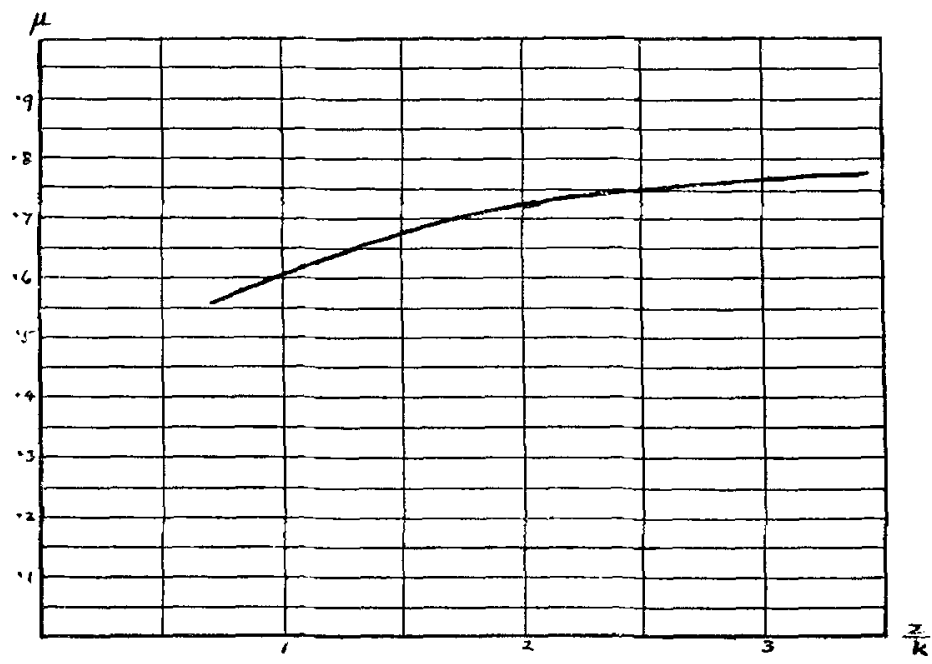

Taking the case in which $e^{2 a}=1$ approximately, so that $\mu$ is independent of $\alpha$, and is given by the curve in fig. 5 in 
terms of $\frac{Z}{k}$, values of $\alpha$ may be deduced by substituting these values of $\mu$ in equation (4). It is interesting to notice that maling $e^{2 a}=1$ in evaluating the ratio $\mu$ involves the assumption that the numbers of negative ions produced by collisions in spaces $S_{1}, S_{2}$, and $S_{3}$ are proportional to the numbers of the original ions, liberated from electrode 2, present in these spaces at any moment of time after the steady state has been reached. Let $\mathrm{N}_{1}, \mathrm{~N}_{2}$, and $\mathrm{N}_{3}$ be these numbers.

Then

$$
u=\frac{n_{2}}{n_{1}+n_{2}+n_{3}}=\frac{\mathrm{N}_{2}}{\mathrm{~N}_{1}+\mathrm{N}_{2}+\mathrm{N}_{3}} \cdot . \cdot .
$$

The values of $\mu$ correspending to a given value of $\frac{Z}{k}$ may be found as follows :

By giving $\xi$ the values $-\cdot 2,-\cdot 1,0, \cdot 1$, and $\cdot 2$ successively the integrals are found to involve only

and

$$
\begin{aligned}
& \int_{0}^{a} d x \int_{0}^{2} z^{-\frac{1}{2}} e^{-10 \cdot 25 \frac{\mathrm{z}}{k} \cdot \frac{x^{2}}{z}} d z, \\
& \int_{0}^{\infty} d x \int_{0}^{2} z^{-\frac{1}{2}} e^{-10 \cdot 25 \frac{\mathrm{z}}{k} \cdot \frac{x^{2}}{z}} d z,
\end{aligned}
$$

where $a$ bas the values $\cdot 4, \cdot 3, \cdot 2$, and $\cdot 1$.

If now $\approx$ is given definite values, e.g. $\cdot 1, \cdot 5,1,1 \cdot 5$, and 2 , the integrals involved are

$$
\int_{0}^{b} e^{-\eta^{2}} d \eta \text { and } \int_{0}^{\infty} e^{-\cdot \eta^{2}} d \eta
$$

where $b$ has certain numerical values.

These integrals can therefore be evaluated by the use of tables. The values of the original integrals after the $\xi$ and $z$ substitutions were plotted against $z$, and the values of the integrals, found by a calculation of areas, were plotted against the corresponding values of $\xi$. The ratio of the new areas gave the value of $\mu$.

When the values of $\mu$ found from the curve in fig. 5 , and the values of $\rho$ taken from the curves in fig. 4 , were sulsstituted in equation (4), the following results were obtained. 
TABLE: I.

\begin{tabular}{|c|c|c|c|c|c|c|c|c|}
\hline \multirow{3}{*}{$\frac{Z}{p}$} & \multicolumn{2}{|c|}{$p=2 \mathrm{~mm}}$. & \multicolumn{2}{|c|}{$p=3 \mathrm{~mm}$} & \multicolumn{2}{|c|}{$p=4 \mathrm{~mm}}$. & \multicolumn{2}{|c|}{$p=5 \mathrm{~mm}}$. \\
\hline & $\mathrm{z}$ & $a$ & $z$ & $a$ & Z & $a$ & $\mathrm{z}$ & $a$ \\
\hline & $k$ & $\vec{p}$ & $\widetilde{k}$ & $\bar{p}$ & $\bar{k}$ & $\bar{p}$ & $\bar{k}$ & $\bar{p}$ \\
\hline 40 & $\ldots$ & $\cdots$ & $\ldots$ & - & $1 \cdot 77$ & .021 & & \\
\hline 45 & $\ldots$ & $\ldots$ & $1 \cdot 42$ & .041 & $1 \cdot 89$ & .044 & $2 \cdot 36$ & 047 \\
\hline 50 & $\cdot 98$ & .065 & $1 \cdot 47$ & .069 & 1.96 & $\cdot 07$ & $2 \cdot 45$ & $\cdot 076$ \\
\hline 55 & 1.01 & $\cdot 101$ & $1 \cdot 515$ & $\cdot 105$ & $2 \cdot 02^{\circ}$ & $\cdot 105$ & $2 \cdot 525$ & $\cdot 11$ \\
\hline 60 & 1.04 & $\cdot 142$ & 1.56 & $\cdot 145$ & $2 \cdot 08$ & $\cdot 149$ & 26 & $\cdot 17$ \\
\hline 70 & 1.09 & $\cdot 25$ & $1 \cdot 64$ & $\cdot 28$ & $2 \cdot 188$ & $\cdot 39$ & & \\
\hline
\end{tabular}

It is observed that for a given value of $\frac{Z}{p}$ the values of $\frac{\alpha}{p}$ increase with the pressure, especially for the larger values of $\alpha$. This is not unexpected, since the relation expressed in (5) must cease to hold when $\alpha$ is large. It is interesting to see how large $\alpha$ may become before this relation ceases to be true (theoretically it is true as long as $\frac{(2 \alpha)^{2}}{L^{2}}+\frac{(2 \alpha)^{3}}{(3}+\ldots$ may be neglected in comparison with $1+2 \alpha$ in the expansion of $e^{2 \alpha}$ ). Now if $\alpha$ is known and substituted in (3) the value of $\mu\left(=\frac{n_{2}}{n_{1}+n_{2}+n_{3}}\right)$ can be evaluated by a method similar to that explained above. In the case of the pressure of $2 \mathrm{~mm}$. the values of $\frac{\alpha}{p}$ were taken from Table I. for three values of $\frac{Z}{p}$. a for each case was substituted in (3) as a first approximation. Values of $\frac{n_{2}}{n_{1}+n_{2}+n_{3}}$ were thus obtained and were compared with the values of $\frac{N_{2}}{N_{1}+N_{2}+N_{3}}$ for the corresponding values of $\frac{Z}{k}$.

TABLE II.

\begin{tabular}{|c|c|c|c|c|c|}
\hline $\mathrm{Z}$ & $\frac{Z}{k}$ & $\frac{\mathrm{N}_{2}}{\mathbf{N}_{1}+\mathbf{N}_{2}+\mathbf{N}_{3}}$ & $\frac{n_{2}}{n_{1}+n_{2}+n_{3}}$ & $\begin{array}{ll}\frac{a}{p} & \text { (1) }\end{array}$ & $\begin{array}{ll}\frac{\alpha}{p} & (2)\end{array}$ \\
\hline 50 & 98 & 605 & 602 & .065 & .065 \\
\hline 60 & $1 \cdot 04$ & .615 & 598 & $\cdot 14$ & $\cdot 134$ \\
\hline 70 & $1 \cdot 09$ & $\cdot 62$ & $\cdot 593$ & .25 & .226 \\
\hline
\end{tabular}


Hence it appears that if $\alpha$ does not exceed the value $\cdot 13$ the relation (5) is very approximately true. The larger values of $\alpha$ given in Table I. are too large, but if these values of $\alpha$ are taken as first approximations to evaluate $\frac{n_{2}}{n_{1}+n_{2}+n_{3}}$ $(\mu)$, and these values substituted in (4), more accurate values of $\alpha$ can be found. The values of $\frac{\alpha}{p}$ thus obtained are given in the last column of Table II., and for the sake of comparison the corresponding values of $\frac{\alpha}{p}$ in Table I. are re
written in the fifth column. written in the fifth column.
The following table gives a comparison of the values of $\frac{\alpha}{p}$ obtained by this method with those found by F.W. Wheatley*, who made use of a more direct method.

TABLE III.

\begin{tabular}{|c|c|c|c|c|c|}
\hline & $\frac{\mathrm{Z}}{p}$ & 40 & 50 & 60 & 70 \\
\hline$\alpha$ & (Wheatley ... & .019 & .055 & $\cdot 118$ & $\cdot 212$ \\
\hline $\bar{p}$ & Smith ......... & $\cdot 021$ & $\cdot 065$ & $\cdot 134$ & $\cdot 2 \cdot 26$ \\
\hline
\end{tabular}

If the values of $\alpha$, for larger values of $\frac{\mathrm{Z}}{p}$, found by Prof. Townsend† are used to evaluate $\mu$ from (3), and $\mu$ is also found by substituting values of $\alpha$ and $\rho$ in (4), another interesting comparison may be made.

Table IV.

\begin{tabular}{|c|c|c|c|}
\hline$\frac{Z}{p}$ & $\frac{\mathbf{Z}}{k}$ & $\mu$ from (3) & $\mu$ from (4) \\
\hline 100 & 1.25 & $\cdot 573$ & 576 \\
\hline$p=2 \mathrm{~mm} .\left\{\begin{array}{l}120 \\
120\end{array}\right.$ & 1.33 & .556 & $\cdot 528$ \\
\hline$p=3 \mathrm{~mm} . \quad 100$ & 1.87 & 606 & .591 \\
\hline
\end{tabular}

* Phil. Mag. Dec. 1913.

$\uparrow$ Phil. Mag. June 1902. 
In the case of $\frac{\mathrm{Z}}{p}=120$, and to a smaller degree in $\frac{\mathrm{Z}}{p}=100$ for $p=3 \mathrm{~mm}$, the positive ions are probably producing ions by collisions to an appreciable extent. This would account for the value of $\mu$, obtained from (4), being smaller than the value found from (3), for expression (4) may be written

$$
\mu=1-\frac{e^{2 \alpha}}{e^{2 \alpha}-1} \cdot \frac{\rho}{1+\rho}
$$

so that

$$
\delta \mu=-\lambda^{2} \delta \rho
$$

and $\delta \rho$ is positive if the positive ions generate ions by collisions.

When the apparatus was tested for small potential differences between the electrodes it was found that in some cases the electrodes 1 and 3 received negative ions. These ions must have diffused to 1 and 3 before they had time to acquire sufficient velocity in the direction of the electric force. The following table shows some of the results obtained for various pressures. V represents the potential difference between the electrodes in volts, $\delta$ the deflexion of the electrometer when connected to the electrodes 1 and 3 , and $l$ the length in $\mathrm{cm}$. of the mean free path of an electron in the gas. The ultra-violet light remained on for one minute in each case, and the deflexion was about 300 when the electrodes 1, 2, and 3 were all connected to the electrometer.

\begin{tabular}{|c|c|c|c|c|c|c|c|c|c|c|c|}
\hline \multicolumn{3}{|c|}{$p=2 \mathrm{~mm} . l=016 \mathrm{~cm}}$. & \multicolumn{3}{|c|}{$p=\cdot 34 \mathrm{~mm} . l=\cdot 1$} & \multicolumn{3}{|c|}{$p=\cdot 044 \mathrm{~mm}, l=\cdot 8$} & \multicolumn{3}{|c|}{$p=017 \mathrm{~mm} . l=2$} \\
\hline V & $\begin{array}{l}\mathrm{Z} \\
p\end{array}$ & $\delta$ & $V$ & $\frac{Z}{p}$ & $\delta$ & $\mathbf{V}$ & $\frac{Z}{p}$ & $\delta$ & $\nabla$ & $\frac{Z}{p}$ & $\delta$ \\
\hline 40 & 10 & -4 & 13 & 20 & -7 & $10 \cdot 5$ & 120 & -8 & $10 \cdot 5$ & 309 & -1 \\
\hline 80 & 20 & 1 & 21 & 32 & -3 & 17 & 193 & -1.5 & 17 & 500 & 0 \\
\hline 120 & 30 & $2 \cdot 5$ & $26 \cdot 5$ & 40 & -1.5 & 21 & 238 & 1.5 & $19 \cdot 5$ & 573 & 75 \\
\hline \multirow[t]{3}{*}{160} & 40 & $9 \cdot 5$ & $30 \cdot 5$ & $45 \cdot 5$ & 0 & 25 & 283 & 8 & 21 & 617 & 2 \\
\hline & & & $34 \cdot 5$ & 52 & 2 & $\ldots$ & $\ldots$ & $\ldots$ & 25 & 735 & 7 \\
\hline & & & 40 & 60 & 6 & & & & & & \\
\hline
\end{tabular}

\section{TABLE V.}

It appears that as the pressure decreases a larger value of $\frac{\mathrm{Z}}{p}$ is necessary to prevent the negative ions from diffusing to the electrodes 1 and 3 . This result is in accordance with 
theory, for a decrease in the pressure of the gas causes an increase in the coefficient of diffusion of the ions into the gas. It seems reasonable to deduce from the above results that, with $\frac{Z}{p}$ not less than 40 , and for pressures of $2,3,4$, and $5 \mathrm{~mm}$., no appreciable number of negative ions can diffuse to the electrodes 1 and 3 .

In conclusion it may be said that no great accuracy is claimed for the values of $\frac{\alpha}{p}$ found in this investigation, but the experiments show directly the production of positive ions by collisions for small values of $\frac{Z}{p}$ and for small potential differences between the electrodes, and also give some idea of the distribution of the current in a gas between parallel plate electrodes.

I desire to express my best thanks to Prof. Townsend, at whose suggestion the investigation was undertaken, for valuable advice given during its progress.

\section{The Theory of Molecular Volumes.} By Gervaise Le Bas, B.Sc.*

\section{PART III.}

Theory of Partial Rings.

The Effect of Unsaturation. Residual Affinity.

(A) $\alpha, \beta$, and $\gamma$ straight Chain Compounds.

QTÄDEL (Ber. xv. p. 2259, 1889) showed that the $\bigcirc \beta$ Halogen compounds always possess smaller volumes than the $\alpha$, and thus we have examples of molecules supposed to be structurally similar possessing different volumes.

The cause of this constitutive peculiarity, for it is undoubtedly such, was not further investigated.

It is a remarkable fact that the $\alpha$ polysubstituted compounds all possess volumes which are comparable to those of monosubstituted compounds in that they follow the additive rule. They are thus normal.

* Communicated by Prof. W. J. Pope, F.R.S. 